\title{
Giant linear plasmids in Streptomyces: a treasure trove of antibiotic biosynthetic clusters
}

\author{
Haruyasu Kinashi
}

Many giant linear plasmids have been isolated from Streptomyces by using pulsed-field gel electrophoresis and some of them were found to carry an antibiotic biosynthetic cluster(s); SCP1 carries biosynthetic genes for methylenomycin, pSLA2-L for lankacidin and lankamycin, and pKSL for lasalocid and echinomycin. Accumulated data suggest that giant linear plasmids have played critical roles in genome evolution and horizontal transfer of secondary metabolism. In this review, I summarize typical examples of giant linear plasmids whose involvement in antibiotic production has been studied in some detail, emphasizing their finding processes and interaction with the host chromosomes. A hypothesis on horizontal transfer of secondary metabolism involving giant linear plasmids is proposed at the end.

The Journal of Antibiotics (2011) 64, 19-25; doi:10.1038/ja.2010.146; published online 8 December 2010

Keywords: antibiotic; biosynthesis; horizontal transfer; linear plasmid; Streptomyces

\section{INTRODUCTION}

Giant linear plasmids are widely distributed in actinobacteria and are involved in secondary metabolism, such as antibiotic production, degradation of aromatic compounds and phytopathogenicity. ${ }^{1-3}$ In particular, the involvement of linear plasmids in antibiotic production was reported in many Streptomyces species. In most cases, antibiotic biosynthetic genes have been studied in detail, but the gross structures of linear plasmids and their structural relationship with linear chromosomes have not, because chemists are mainly interested in biosynthetic mechanism. However, accumulated data suggest that giant linear plasmids have played critical roles in genome evolution and horizontal transfer of secondary metabolism. Thus, to clarify the mechanism of these biologically important phenomena, precise structural and sequence comparison of linear plasmids and chromosomes is necessary. I have been in Professor Hutchinson's laboratory, Wisconsin University, from 1984 to 1986 and studied biosynthesis of the polyether antibiotic lasalocid in Streptomyces lasaliensis. This experience after returning to Japan resulted in the discovery of giant linear plasmids from many antibiotic-producing Streptomyces species, including pKSL from S. lasaliensis. ${ }^{4,5}$ In this review, I summarize giant linear plasmids involved in antibiotic production in Streptomyces, emphasizing their finding processes and interaction with the host chromosomes. At the end, possible mechanisms on how giant linear plasmids could function in horizontal transfer of secondary metabolism, are discussed.

\section{SCP1 IN STREPTOMYCES COELICOLOR}

Isolation and characterization of the giant linear plasmid of SCP1 It has long been suggested that plasmids are involved in antibiotic production in Streptomyces species, on the basis of genetic instability of the antibiotic-producing ability. ${ }^{6}$ In particular, extensive genetic studies by Hopwood and colleagues ${ }^{7,8}$ confirmed that the plasmid SCP1 carries the biosynthetic (mmy) genes for methylenomycin (Figure 1) in S. coelicolor A3(2). Furthermore, the mmy gene cluster was cloned by mutational cloning and analyzed in detail, ${ }^{9}$ even though SCP1 itself was still resistant to isolation and its physical identity had not been clarified. The application of pulsed-field gel electrophoresis (PFGE) to Streptomyces DNA changed this situation and revealed that SCP1 is a giant linear plasmid of about $350 \mathrm{~kb} .{ }^{10}$ At the same time, many linear plasmids were detected in other antibiotic-producing strains, including S. rochei and S. lasaliensis (Table 1). ${ }^{4}$ Furthermore, subsequent physical analysis revealed that Streptomyces chromosomes themselves are linear. ${ }^{11,12}$

Restriction analysis and treatment with exonuclease III and $\lambda$ exonuclease showed that SCP1 contains terminal inverted repeats (TIRs) of about $80 \mathrm{~kb}$ at both ends and the $5^{\prime}$ ends are blocked by a terminal protein (Figure 2a). ${ }^{13}$ These two features proved to be conserved in most of linear plasmids and chromosomes in actinobacteria. Cloning and sequencing of the terminal fragments revealed that the telomere sequence of SCP1 could not form a Y-shaped foldback structure, ${ }^{14}$ a feature conserved in most Streptomyces linear replicons, ${ }^{15}$ which may function in terminal replication. ${ }^{16}$ However,

Department of Molecular Biotechnology, Graduate School of Advanced Sciences of Matter, Hiroshima University, Higashi-Hiroshima, Japan Correspondence: Dr H Kinashi, Department of Molecular Biotechnology, Graduate School of Advanced Sciences of Matter, Hiroshima University, 1-3-1 Kagamiyama, Higashi-Hiroshima 739-8530, Japan.

E-mail: kinashi@hiroshima-u.ac.jp

Dedicated to the late $\operatorname{Dr} \mathrm{C}$ Richard Hutchinson for his exceptional contributions to natural product biosynthesis, engineering and drug discovery.

Received 15 September 2010; revised 1 November 2010; accepted 3 November 2010; published online 8 December 2010 


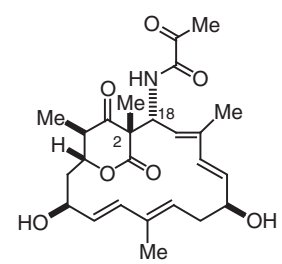

ankacidin C

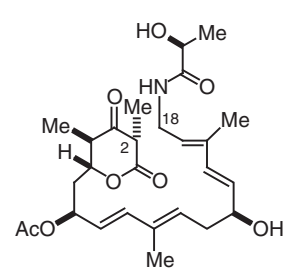

LC-KA05

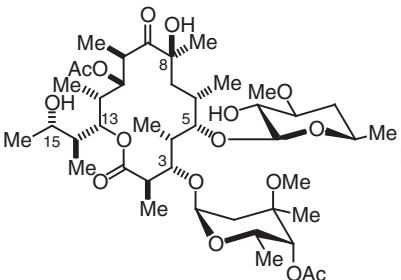

lankamycin

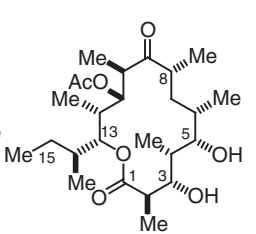

8,15-dideoxylankanolide

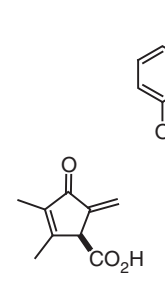

methylenomycin<smiles>COC1=C(C(N)=O)C(=O)[C@@]2(O)C(O)=C3C(=O)c4c(O)cccc4[C@@](C)(O)[C@H]3[C@@H](O)[C@@H]2[C@H]1OC</smiles>

oxytetracycline

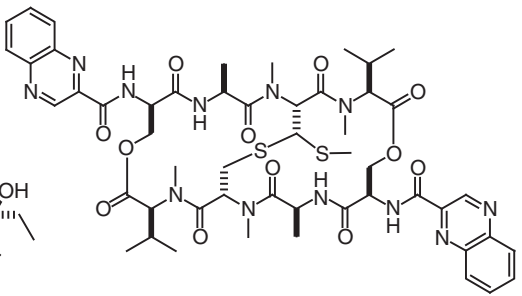

echinomycin

Figure 1 Structures of linear plasmid-determined antibiotics and some of their intermediates.

Table 1 Giant linear plasmids in antibiotic-producing Streptomyces strains

\begin{tabular}{|c|c|c|c|}
\hline Strains & Antibiotics & Sizes of plasmids (kb) & References \\
\hline S. lasaliensis NRRL 3382R & Lasalocid, echinomycin & 520 (pKSL) & $4,5,57,59$ \\
\hline S. coelicolor A3(2) 1147 & Methylenomycin & $356(\mathrm{SCP} 1)$ & $10,13,14,17$ \\
\hline S. violaceoruber JCM 4979 & Methylenomycin & plasmid ladder (390-630) & 4,10 \\
\hline \multirow[t]{2}{*}{ S. rochei 7434AN4 } & Lankacidin & 17 (pSLA2-S), 100 (pSLA2-M) & $4,42,43$ \\
\hline & Lankamycin & $210(p S L A 2-L)$ & \\
\hline S. rimosus MV17 & Oxytetracycline & 1000 & 60,61 \\
\hline S. clavuligerus IFO 13307 & Cephamycin, clavulanic acid & 12 (pSCL1), 150 (pSCL2) & $63-66$ \\
\hline NRRL 3585 & & $450(\mathrm{pSCL} 3)$ & \\
\hline S. clavuligerus ATCC 27064 & Many antibiotics & $1800(\mathrm{pSCL} 4)$ & 67 \\
\hline S. carzinostaticus ATCC 15944 & Neocarzinostatin & 880 & 70 \\
\hline S. atroolivaceus S-140 & Leinamycin & 900 & 71 \\
\hline S. globisporus & C-1027 & 160 & 72 \\
\hline
\end{tabular}

a

SCP1
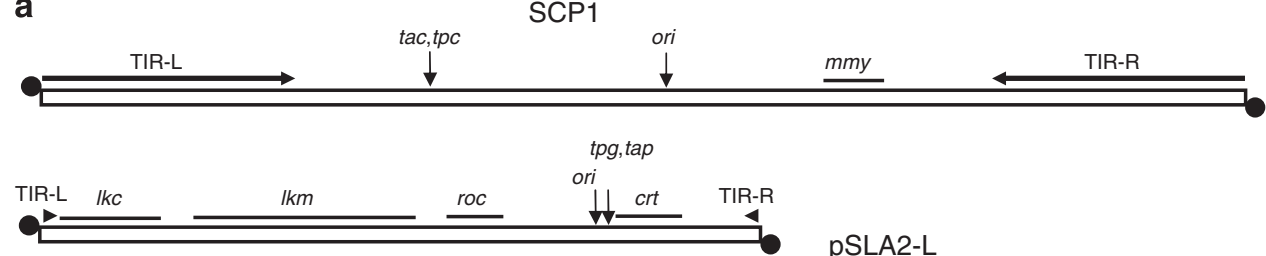

pSLA2-L

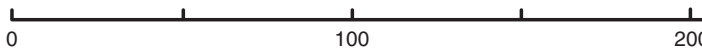

1

$300 \mathrm{~kb}$

b

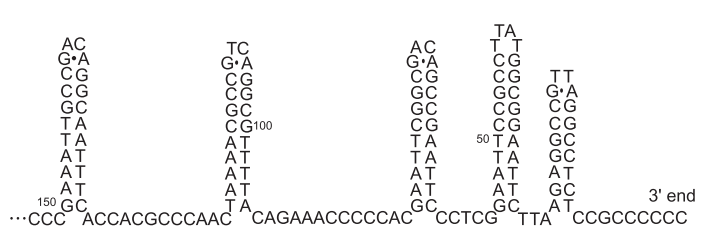

SCP1

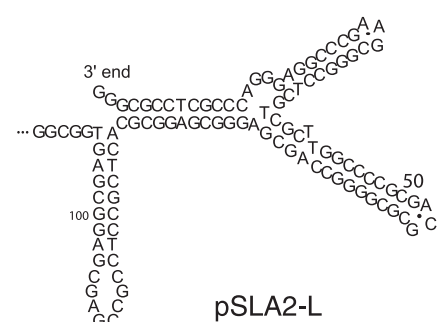

Figure 2 The gross structures of the linear plasmids, SCP1 and PSLA2-L (a), and possible secondary telomere structures deduced from their sequences (b). 

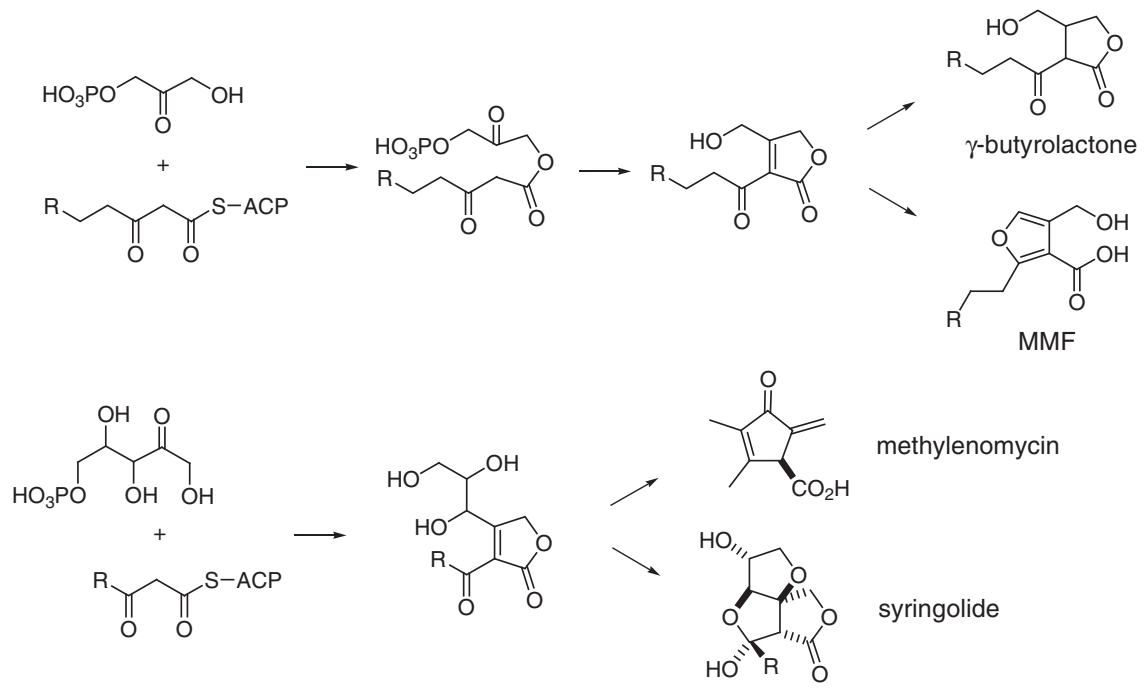

Figure 3 Similar biosynthetic pathways for $\gamma$-butyrolactone, methylenomycin furan, methylenomycin and syringolide.

several hairpin loop structures are still possible to be made (Figure 2b). Eventually, the complete nucleotide sequence (356 $023 \mathrm{bp}$ ) of SCP1 was determined, ${ }^{17}$ which confirmed the size of TIRs (75 $122 \mathrm{bp)}$ and located the mmy gene cluster, a replication origin, two partition genes (parA and parB) and others. However, a terminal protein gene $(t p g)^{18}$ was not identified from the sequence data, because of the unique telomere sequence of SCP1. Later, Huang et al. ${ }^{19}$ isolated the terminal protein as a complex with SCP1 and finally identified the $t p c$ (terminal protein of SCP1, orf127) and tac (telomere-associated protein of SCP1, orf125) genes of SCP1.

Biosynthesis of methylenomycin and autoregulators in S. coelicolor In silico analysis of the SCP1 sequence together with gene disruption experiments identified the $19-\mathrm{kb} m m y$ gene cluster consisting of 21 genes from $m m y R$ to $m m y F^{20}$ It is noteworthy that a new type of autoregulators, 2-alkyl-4-hydroxymethylfuran-3-carboxylic acids named methylenomycin furans (MMFs) (Figure 3), were isolated from $S$. coelicolor, ${ }^{21}$ in addition to $\mathrm{SCB} 1,{ }^{22}$ a typical $\gamma$-butyrolactone autoregulator. Thus, S. coelicolor produces two different types of autoregulators; MMFs stimulates methylenomycin production, ${ }^{20}$ whereas SCB1 stimulates the biosynthesis of an unknown polyketide. ${ }^{23}$ Three genes in the $m m y$ gene cluster, $m m f L, m m f H$ and $m m f P$, constitute a biosynthetic cluster for MMFs. $^{20}$ The $m m f L$ gene product shows $27 \%$ identity with AfsA, a key enzyme for A-factor biosynthesis in S. griseus, ${ }^{24}$ whereas $s c b A$, another homologue of $a f_{S} A$ for SCB1 synthesis, is located on the chromosome. ${ }^{25}$ The same precursors, dihydroxyacetone phosphate and $\beta$-ketoacyl CoA, were incorporated into both types of autoregulators. $^{26,27}$ Thus, $\gamma$-butyrolactones and MMFs are biosynthesized by identical butenolide intermediates, followed by reduction of a double bond ( $\gamma$-butyrolactone) or recyclization of a butenolide ring (MMF) (Figure 3).

The incorporation pattern of $\left[\mathrm{U}^{13} \mathrm{C}\right]$ glycerol suggests that methylenomycin itself is also synthesized by a butenolide intermediate, which is formed by condensation of pentulose-5-phosphate with $\beta$-ketoacyl CoA (Figure 3). ${ }^{28}$ It is noteworthy that the same butenolide compound has been proposed as an intermediate for syringolide biosynthesis in Pseudomonas syringae. ${ }^{29}$
Interaction of SCP1 with S. coelicolor chromosome and other replicons SCP1 is known to interact with the S. coelicolor chromosome to generate various SCP1-chromosome hybrid structures. A 1850-kb linear DNA was found in S. coelicolor 2106, a cysD donor, which transfers a $c y s D$ phenotype in the mating with an SCP1-free strain. ${ }^{30}$ It was revealed that this large linear DNA and the 7.2-Mb linear chromosome in strain 2106 were formed by a single crossover of SCP1 and the wild-type chromosome concomitant with short deletions. ${ }^{31}$ As the telomere sequences of SCP1 and the host chromosome are totally different, both chimeric linear DNAs do not have TIRs. The $1850-\mathrm{kb}$ DNA could not be cured by various mutagenic treatments. Thus, we concluded that the $7.2-\mathrm{Mb}$ chromosome and the $1850-\mathrm{kb}$ DNA are chimeric linear chromosomes and were named chromosomes I and II, respectively. The recombination event occurred in strain 2106 may be considered as a model for duplication and multiplication of a linear chromosome. ${ }^{3,31}$

It is also known that SCP1 is integrated in the central region or in other regions of the host chromosome. In the mating with SCP1-free partners, these SCP1-integrated strains showed either a bidirectional or unidirectional gradient of transfer of genetic markers with respect to the SCP1 integration site. The integrated structures of SCP1 in an NF (normal fertility) strain $2612^{32}$ and an NF-like strain A634 ${ }^{33}$ were analyzed. Although both strains show a bidirectional gene transfer, the direction of SCP1 insertion and the deletions at both sides of SCP1 were different. ${ }^{34,35}$ Thus, several rounds of recombination might have occurred at both sides of SCP1 to generate the present integrated forms of SCP1 in these strains.

S. violaceoruber JCM 4979, another methylenomycin producer, carries series of linear plasmids ranging from 390 to $630 \mathrm{~kb}$ with a size difference of about $35 \mathrm{~kb}$ (Table 1 ). ${ }^{4}$ Restriction and sequencing analysis suggested that these linear plasmids were generated by insertion of a 31-kb circular plasmid SCP2 into SCP1 by homologous recombination between two copies of transposon Tn5714 on each plasmid, followed by amplification of a 36-kb segment comprising of adjacent SCP1 and SCP2 regions (Kinashi et al. ${ }^{36}$ and unpublished results).

On the other hand, S. violaceoruber SANK 95570, from which Haneishi et $a l .{ }^{37}$ first isolated methylenomycin, carries a $163-\mathrm{kb}$ circular plasmid pSV1. It was revealed that pSV1 and SCP1 have 
homology to each other only around the $m m y$ and par $A, B$ regions. ${ }^{38}$ This result eliminated models in which pSV1 was generated by circularization of SCP1 or vice versa. It was rather suggested that the $m m y$ gene cluster was horizontally transferred as a set together with the parA, $B$ region from $\mathrm{pSV} 1$ to SCP1. The truncated gene (SCP1.216Ac) at the left side of the mmy-parAB segment on SCP1 might have been generated by non-homologous recombination (see later).

\section{PSLA2-L IN S. ROCHEI}

\section{Isolation and characterization of pSLA2-L}

Hayakawa et al. ${ }^{39}$ first isolated a linear plasmid pSLA2 from $S$. rochei 7434AN4, which produces two structurally unrelated polyketide antibiotics, lankacidin and lankamycin (Figure 1). Physical analysis revealed that pSLA2 is $17 \mathrm{~kb}$ in size and is structurally similar to adenovirus and bacteriophage $\phi 29$ DNA; TIRs are present at both ends and a terminal protein is bound to the $5^{\prime}$ ends. ${ }^{40,41}$ However, it was later found that pSLA2 was not involved in the production of lankacidin or lankamycin.

The application of PFGE to strain 7434AN4 revealed two linear plasmids, pSLA2-L and pSLA2-M, in addition to pSLA2-S (=pSLA2 $)^{4}$ (Table 1). A complete correlation was observed between the presence of PSLA2-L and the production of lankacidin and lankamycin in mutants with a different plasmid profile. ${ }^{42}$ Finally, nucleotide sequencing of pSLA2-L (210614 bp) revealed the GC content $(72.8 \%)$, the size of TIRs (1992 bp) and 143 open reading frames $(\text { orfs })^{43}$ (Figure 2a). pSLA2-L has a conserved telomere sequence, which can form a typical Y-shaped foldback structure (Figure $2 b$ ). ${ }^{44}$

Homology searching of the 143 Orfs along with gene disruption experiments delimited the ranges of the biosynthetic clusters for lankacidin ( $l k c$, orf4-orf18), lankamycin ( $l k m$, orf24-orf53), a cryptic type-II polyketide (roc, orf62-orf70) and carotenoid (crt, orf104orf110). ${ }^{43}$ In addition, many regulatory genes were found; a $\gamma$-butyrolactone biosynthetic gene $(\operatorname{srr} X, \underline{S}$. rochei regulatory gene $\mathrm{X})$, six tetR family repressor genes ( $\operatorname{sr} A, \bar{s} r \bar{B}, \operatorname{srr} C, \operatorname{srrD}, \operatorname{srrE}$, and $s r r F)$ including the $\gamma$-butyrolactone receptor gene (srrA) and three Streptomyces antibiotic regulatory protein (SARP) genes (srrW, srrY and $s r r Z$ ). Thus, two-thirds of pSLA2-L DNA is occupied by secondary metabolism-related genes.

\section{The regulatory cascade for secondary metabolism}

Disruption of the regulatory genes indicated that $\operatorname{srr} X$ has a positive effect on the production of lankacidin and lankamycin, and a negative effect on spore formation. ${ }^{45}$ This effect is different from that of $a f_{s} A$ in S. griseus, which shows positive effects on both streptomycin production and spore formation. ${ }^{46}$ The receptor gene srrA has counteracting functions against both effects of $\operatorname{sr} X$. On the other hand, $\operatorname{srr} B$ has a negative effect only on antibiotic production, whereas $\operatorname{sr} C$ has a positive effect on spore formation without affecting antibiotic production. Extensive transcriptional analysis revealed the main signaling pathway; $s r r X \rightarrow s r r A \rightarrow s r r Y$-srrC $\rightarrow$ srr $Z \rightarrow l k m A I$ (PKS gene) $\rightarrow$ lankamycin production. ${ }^{47,48}$ The SARP gene $\operatorname{sr} Y$ also activates lankacidin production through an unidentified pathway. We speculate that the cistron organization of $\operatorname{srr} Y$-srrC functions as a switch from antibiotic production to spore formation. The expression of $\operatorname{srr} Y$-srrC activates antibiotic production, whereas its cessation may turn the phase to spore formation. However, the details of the regulatory pathway downstream of $\operatorname{srC}$ have not been clarified.

\section{Biosynthesis of lankacidin and lankamycin}

Lankacidin C is a 17 -membered macrocyclic antibiotic (Figure 1), different from usual even-membered macrolides like erythromycin.
This unique structure and the gene organization of the lankacidin synthase $(l k c)$ cluster raised two interesting questions. (i) How is the carbon-carbon linkage formed between $\mathrm{C}_{2}$ and $\mathrm{C}_{18}$. (ii) How can five ketosyntase domains in the $l k c$ cluster accomplish eight condensation reactions necessary for lankacidin synthesis. The amine oxidase $(l k c E)$ disruptant accumulated an acyclic intermediate LC-KA05 (Figure 1), which was converted to lankacidin C by other mutants. ${ }^{49}$ This result indicates that LkcE oxidizes LC-KA05 to an unisolated imine $\left(\mathrm{C}_{18}=N\right)$, which in turn receives a nucleophilic attack by the enolic carbon $\left(\mathrm{C}_{2}\right)$ to form the $\mathrm{C}_{2}-\mathrm{C}_{18}$ linkage. In regard to the second question, we proposed a hypothesis of modular-iterative mixed polyketide biosynthesis. ${ }^{49}$ Although we isolated no confirmative metabolites, heterologous expression of the $l k c$ cluster $(l k c A-l k c O)$, gene fusion of $l k c F$ and $l k c G$, and extensive disruption of the $l k c$ genes supported this hypothesis. ${ }^{50,51}$ Thus, LkcC may be used iteratively (four times) and LkcA, LkcF and LkcG modularly to accomplish eight condensation reactions for the lankacidin skeleton.

The 14-membered macrolide antibiotic, lankamycin contains a 3-hydroxy-2-butyl group at C-13 (Figure 1), which is different from an ethyl group in erythromycin. The feeding experiment of $\left[3-{ }^{2} \mathrm{H}\right]-$ isoleucine indicates that isoleucine was oxidized to 2-methylbutyrate and incorporated as a starter. ${ }^{52}$ Thus, a colinear relationship was conserved here between the $l \mathrm{~km}$-PKS domains and biosynthetic reactions. On the basis of the structures of intermediates accumulated by mutants of the P450 hydroxylase and glycosyl transferase genes, a possible post-PKS pathway was proposed; 8,15-dideoxylankanolide (Figure 1) $\rightarrow$ 8-deoxylankanolide $\rightarrow$ 3-O-L-arcanosyl-8-deoxylankanolide $\rightarrow$ 3-O-L-arcanosyl lankanolide $\rightarrow 3-\mathrm{O}$-L-arcanosyl-5-O-D-chalcosyl lankanolide $\rightarrow$ lankamycin. ${ }^{52,53}$

\section{Synergistic action of lankacidin and lankamycin}

The close location of the lankacidin and lankamycin clusters on pSLA2-L and the co-regulation of their biosynthesis by the same unisolated $\gamma$-butyrolactone compound suggest that two antibiotics may have synergistic action. ${ }^{2}$ This idea proved to be true by a collaborative work with Drs Yonath and Mankin. ${ }^{54}$ Furthermore, crystallographic analysis and chemical probing showed that two antibiotics were bound to a different ribosome site, lankacidin to the peptidyl transferase center and lankamycin to the nascent peptide exit tunnel, leading to a synergistic inhibition of peptide formation. A similar super-cluster for cephamycin and clavulanic acid was found in $S$. clavuligerus, ${ }^{55}$ where the two metabolites function synergistically as an antibiotic and a $\beta$-lactamase inhibitor, respectively, and their biosynthesis is regulated by the SARP gene $c c a R .^{56}$

\section{PKSL IN S. LASALIENSIS}

As described above, we investigated lasalocid biosynthesis in S. lasaliensis NRRL 3382R in Professor Hutchinson's laboratory. Although we constructed many lasalocid-nonproducing mutants, cosynthesis experiments with many combinations, all failed to give positive-lasalocid production. ${ }^{5}$ This result suggested that all of the mutants might have the same genetic defect in antibiotic production, for example, the loss of a plasmid carrying the biosynthetic gene cluster for lasalocid. The antibiotic-producing ability was transferred by protoplast fusion from producers to non-producers, supporting this hypothesis. However, we could not detect such a plasmid with conventional agarose gel electrophoresis.

A $520-\mathrm{kb}$ linear plasmid pKSL was finally detected by PFGE analysis, ${ }^{4}$ its presence and an antibiotic-producing ability being correlated in protoplast fusants. ${ }^{5}$ The cosmid library of S. lasaliensis 
ATCC 35852 (=NRRL 3382R) was constructed and screened with a ketosynthase probe, which afforded three overlapping cosmids. Their shotgun sequencing revealed an $82-\mathrm{kb}$ region covering the entire lasalocid biosynthetic $(l s d)$ cluster. ${ }^{57}$ It is interesting that the $l s d$ cluster contains a gene set for producing ethylmalonyl-CoA, different from other polyether clusters. This may be reasonable, because the biosynthesis of lasalocid requires three moles of ethylmalonyl-CoA (Figure 1). Although the disruption of the $l s d$ genes has not been succeeded, the enzymatic activity of the epoxide hydrolase Lsd19 was confirmed by in vitro formation of lasalocid from a synthesized bisepoxide through cascade epoxide opening reactions. ${ }^{58}$ The whole gene cluster for echinomycin biosynthesis was cloned and expressed in E. coli ${ }^{59}$ which also confirmed the function of the echinomycin cluster on pKSL.

\section{LINEAR PLASMIDS IN S. RIMOSUS}

S. rimosus R6, an oxytetracycline producer, carries pPZG101, a giant linear plasmid of $387 \mathrm{~kb}$, which has a unique central region of $30 \mathrm{~kb}$ flanked by inverted repeats of about $180 \mathrm{~kb} .{ }^{60}$ During the study of genetic instability of S. rimosus R6, one overproducer (MV17) was found to carry a $1-\mathrm{Mb}$ linear plasmid, pPZG103. This plasmid arose from a single crossover between pPZG101 and the chromosome, such that about $200 \mathrm{~kb}$ at one end is derived from pPZG101 and the other $800 \mathrm{~kb}$ is the chromosomal terminal sequence carrying the oxytetracycline cluster. ${ }^{61}$ The chromosome had a parental structure, so there was an increased copy number of the oxytetracycline cluster due to the extra copy on the plasmid.

\section{LINEAR PLASMIDS IN S. CLAVULIGERUS}

$\beta$-Lactams are produced by a wide range of microorganisms including fungi and gram-positive and negative bacteria. $\beta$-Lactam biosynthetic genes form a cluster in which individual genes and their organizations are conserved beyond the barrier between prokaryote and eukaryote. From these results, horizontal transfer of the $\beta$-lactam cluster was proposed. $^{62}$

Screening with PFGE for linear plasmids in $\beta$-lactam producing actinomycete strains was carried out by us and Roy's group, which revealed linear plasmids of $12-450 \mathrm{~kb}$. Among them, S. clavuligerus NRRL 3585 (=IFO 13307), a producer of cephamycin and clavulanic acid, carried three linear plasmids, pSCL1 (12 kb), pSCL2 (120 kb) and pSCL3 $(430 \mathrm{~kb}) .{ }^{63,64}$ However, none of them hybridized to the biosynthetic probes, the $p c b C$ gene for isopenicillin synthase or the $c e f D$ and $c e f E$ genes for isopenicillin $\mathrm{N}$ epimerase and deacetoxycephalosporin C synthase, respectively. Nucleotide sequencing of pSLC1 and pSLC2 neither revealed any genes for cephamycin or clavulanic acid biosynthesis. ${ }^{65,66}$

Recently, Madema et al. ${ }^{67}$ isolated an extremely large linear plasmid, pSCL4, from S. clavuligerus ATCC 27064 and determined its $1.8-\mathrm{Mb}$ nucleotide sequence. Surprisingly, this plasmid was densely packed with an exceptionally large number of gene clusters for the potential production of antibiotics, such as staurosporine, moenomycin, $\beta$-lactams and enediynes. Although strain ATCC 27064 was originally identical with strains IFO 13307 and NRRL 3585, it does not carry pSCL1, pSCL2, or pSCL3. Instead, its on going genome project revealed that only a 7-kb DNA in the assembly matched the pSCL1 sequence. Thus, it was suggested that pSCL4 was formed through recombination of pSCL1 with the arms of the host chromosome. The authors claimed that pSCL4 is a plasmid, but not a chromosome, on the basis of the fact that it does not code any essential genes for survival. This idea should be proved experimentally by curing of pSCL4 as well as pPZG103 in S. rimosus.

\section{OTHER GIANT LINEAR PLASMIDS}

The first application of PFGE to Streptomyces DNA enabled the detection of giant linear plasmids in additional antibiotic-producing Streptomyces strains; S. fradiae C373.1 (tylosin), S. parvulus JCM 4068 (actinomycin) and S. venezuelae JCM 4526 (chloramphenicol). ${ }^{4}$ Since then, many giant linear plasmids have been isolated from antibiotic producers, for example, pSB1 from $S$. bambergiensis (moenomycin), ${ }^{68}$ and pSA1 and pSA2 from S. avermitilis (avermectin). ${ }^{69}$ However, their functions in antibiotic production have not been confirmed. In contrast, to our surprise Shen et al. (B. Shen, personal communication) recently found that the biosynthetic gene clusters for neocarzinostatin in S. carzinostaticus, ${ }^{70}$ leinamycin in S. globispora ${ }^{71}$ and C-1027 in S. atroolivaceus, ${ }^{72}$ are all located on a giant linear plasmid (Table 1). The same situation may be present in other cases, where the antibiotic clusters have not been located in spite of extensive biosynthetic studies. Therefore, the analysis with PFGE and Southern hybridization will increase the number of giant linear plasmids involved in antibiotic production.

\section{HORIZONTAL TRANSFER OF ANTIBIOTIC PRODUCTION}

As described above, giant linear plasmids are widely distributed in Streptomyces and may be contributing to horizontal transfer of antibiotic production, directly and indirectly. First, the conjugal transfer of linear plasmids carrying an antibiotic cluster transfers a producing ability to the accepted strains. Ravel et al. ${ }^{73}$ reported that the giant linear plasmids, pRJ3L (322 kb) and pRJ28 (330 kb), both encoding mercury resistance, were successfully transferred by conjugation from their streptomycete hosts to S. lividans.

Second, single crossover between linear plasmids and linear chromosomes causes end exchange, which transfers the antibiotic cluster from the plasmid to the chromosome or vice verse. As described above, the methylenomycin cluster was transferred from SCP1 to the $S$. coelicolor chromosome ${ }^{31}$ and the oxytetracycline cluster from the $S$. rimosus chromosome to pPGZ3. ${ }^{61}$ The linear plasmid SLP2 in S. coelicolor also proved to be a composite of the chromosome end and a linear plasmid, ${ }^{74}$ although antibiotic production was not involved in this case. Comparison of the genome sequences of $S$. coelicolor, S. avermitilis and $S$. ambofaciens revealed that the central parts of the chromosomes are highly syntenic, whereas the terminal regions are species-specific. ${ }^{75}$ This fact may also reflect end exchange of Streptomyces linear chromosomes. As essential genes are not coded in the terminal regions, they are exchangeable except for the extreme ends that are necessary for terminal replication. Even the extreme ends are exchangeable, as long as the machinery for terminal replication are linked to new ends.

Third, more complex interactions between linear plasmids and chromosomes were observed. In S. coelicolor, SCP1 is integrated in the central regions of the chromosome with different sizes of deletions at both sides. ${ }^{34,35}$ pSCL4 of $S$. clavuligerus is composed of a $7-\mathrm{kb}$ pSCL1 sequence flanked by the two chromosomal arms. ${ }^{67}$ In this connection, I discussed on horizontal transfer of the extremely condensed biosynthetic clusters on pSLA2-L. ${ }^{3}$ We could not detect any transposition-related genes or sequences surrounding the clusters, except for several truncated genes. ${ }^{43}$ This is true for the methylenomycin cluster on SCP $1 .{ }^{17,20}$ On the basis of these results, I hypothesized that in most cases horizontal transfer has not occurred by a direct transposition of the cluster, but has involved multiple recombination events. Several cycles of single crossover at both sides of the cluster generate a gene organization that gives a probably misleading impression of having been generated by a direct transposition of the 


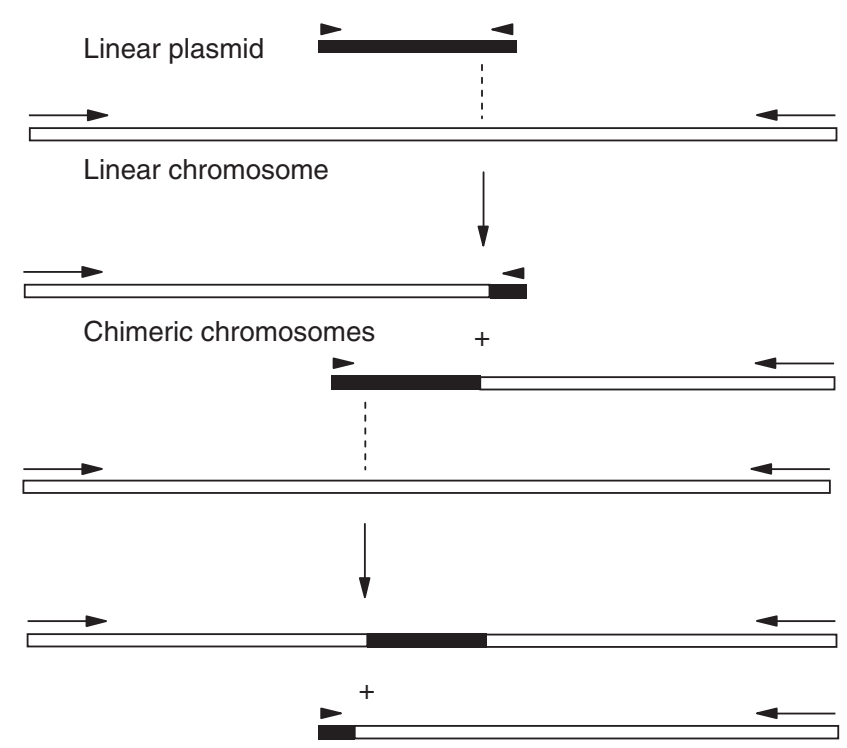

Figure 4 Recombination of linear replicons, which could cause end exchange and plasmid integration.

cluster. Truncated genes frequently found around the cluster may be traces of non-homologous recombination.

The same mechanism could be applied to horizontal transfer of much larger linear plasmids. The fact that two chimeric chromosomes are stably maintained in S. coelicolor strain 2106 (see ref. $^{31}$ ) indicates that single crossover occurs even in more central regions of chromosome. Nevertheless, chimeric linear replicons without TIRs may have somewhat inferior properties, because most of Streptomyces linear replicons have TIRs. For example, linear replicons without TIRs cannot take a racket frame structure, ${ }^{76}$ which might function in terminal replication or intramolecular replicational repair of terminal deletions. ${ }^{3,31}$ Thus, chimeric DNAs without TIRs generated by single crossover might be followed by second and third homologous or nonhomologous recombinations to recover TIRs. These series of recombination events result in a structure, which looks like a product of direct integration of a linear plasmid (Figure 4). Precise sequence comparison of giant linear plasmids and linear chromosomes on the basis of their complete sequencing will finally clarify the mechanism of horizontal transfer of secondary metabolism.

\section{ACKNOWLEDGEMENTS}

I would like to thank many researchers who contributed to our works described in this review, especially, Drs K Sakaguchi, CR Hutchinson, DA Hopwood, KF Chater, T Hanafusa, M Yamasaki, M Redenbach, K Hiratsu, S Mochizuki and K Arakawa. I also would like to thank Dr B Shen for unpublished results.

1 Kinashi, H. Linear plasmids from actinomycetes. Actinomycetol. 8, 87-96 (1994).

2 Chater, K. F. \& Kinashi, H. Streptomyces linear plasmids: their discovery, functions, interactions with other replicons, and evolutionary significance. in Microbiology Monographs, Vol. 7, Microbial Linear Plasmids (eds Meinhardt, F. \& Klassen, R.) 1-31 (Springer, Berlin/Heidelberg, Germany, 2007).

3 Kinashi, H. Antibiotic production, linear plasmids and linear chromosomes in Streptomyces. Actinomycetol 22, 20-29 (2008).

4 Kinashi, H. \& Shimaji, M. Detection of giant linear plasmids in antibiotic producing strains of Streptomyces by the OFAGE technique. J. Antibiot. 40, 913-916 (1987).

5 Kinashi, H., Otten, S. L., Duncan, J. S. \& Hutchinson, C. R. Frequent loss and restoration of antibiotic production by Streptomyces lasaliensis. J. Antibiot. 41, 624-637 (1988).
6 Okanishi, M., Ita, T. \& Umezawa, H. Possible control of formation of aerial mycelium and antibiotic production in Streptomyces by episomic factors. J. Antibiot. 23, 45-47 (1970).

7 Kirby, R., Wright, L. F. \& Hopwood, D. A. Plasmid-determined antibiotic synthesis and resistance in Streptomyces coelicolor. Nature 254, 265-267 (1975).

8 Kirby, R. \& Hopwood, D. A. Genetic determination of methylenomycin synthesis by the SCP1 plasmid of Streptomyces coelicolor A3(2). J. Gen. Microbiol. 98, 239-252 (1977).

9 Chater, K. F. \& Bruton, C. J. Resistance, regulatory and production genes for the antibiotic methylenomycin are clustered. EMBO J. 4, 1893-1897 (1985).

10 Kinashi, H., Shimaji, M. \& Sakai, A. Giant linear plasmids in Streptomyces which code for antibiotic biosynthesis genes. Nature 328, 454-456 (1987).

11 Lin, Y. S., Kieser, H. M., Hopwood, D. A. \& Chen, C. W. The chromosomal DNA of Streptomyces lividans 66 is linear. Mol. Microbiol. 10, 923-933 (1993).

12 Lezhava, A. et al. Physical map of the linear chromosome of Streptomyces griseus. J. Bacteriol. 177, 6492-6498 (1995).

13 Kinashi, H. \& Shimaji-Murayama, M. Physical characterization of SCP1, a giant linear plasmid from Streptomyces coelicolor. J. Bacteriol. 173, 1523-1529 (1991).

14 Kinashi, H., Shimaji-Murayama, M. \& Hanafusa, T. Nucleotide sequence analysis of the unusually long terminal inverted repeats of a giant linear plasmid, SCP1. Plasmid 26, 123-130 (1991).

15 Huang, C. H., Lin, Y. S., Yang, Y. L., Huang, S. W. \& Chen, C. W. The telomeres of Streptomyces chromosomes contain conserved palindromic sequences with potential to form complex secondary structures. Mol. Microbiol. 28, 905-916 (1998).

16 Qin, Z. \& Cohen, S. N. Replication at the telomeres of the Streptomyces linear plasmid pSLA2. Mol. Microbiol. 28, 893-903 (1998).

17 Bentley, S. D. et al. SCP1, a 356,023 bp linear plasmid adapted to the ecology and developmental biology of its host, Streptomyces coelicolor A3(2). Mol. Microbiol. 51, 1615-1628 (2004).

18 Bao, K. \& Cohen, S. N. Terminal proteins essential for the replication of linear plasmids and chromosomes in Streptomyces. Genes Dev. 15, 1518-1527 (2001).

19 Huang, C. H. et al. The telomere system of the Streptomyces linear plasmid SCP1 represents a novel class. Mol. Microbiol. 63, 1710-1718 (2007).

20 O'Rourke, S. et al. Extracellular signalling, translational control, two repressors and an activator all contribute to the regulation of methylenomycin production in Streptomyces coelicolor. Mol. Microbiol. 71, 763-778 (2009).

21 Corre, C. Song, L., O'Rourke, S., Chater, K. F. \& Challis, G. L. 2-Alkyl-4-hydroxymethylfuran-3-carboxylic acids, antibiotic production inducers discovered by Streptomyces coelicolor genome mining. Proc. Natl Acad. Sci. USA. 105, 17510-17515 (2008).

22 Takano, E. et al. Purification and structural determination of SCB1, a $\gamma$-butyrolactone that elicits antibiotic production in Streptomyces coelicolor A3(2). J. Biol. Chem. 275, 11010-11016 (2000)

23 Takano, E. et al. A bacterial hormone (the SCB1) directly controls the expression of a pathway-specific regulatory gene in the cryptic type I polyketide biosynthetic gene cluster of Streptomyces coelicolor. Mol. Microbiol. 56, 465-479 (2005).

24 Horinouchi, S., Kumada, Y. \& Beppu, T. Unstable genetic determinant of A-factor biosynthesis in streptomycin-producing organisms: cloning and characterization. J. Bacteriol. 158, 481-487 (1984).

25 Takano, E., Chakraburtty, R., Nihira, T., Yamada, Y. \& Bibb, M. J. A complex role for the $\gamma$-butyrolactone SCB1 in regulating antibiotic production in Streptomyces coelicolor A3(2). Mol. Microbiol. 41, 1015-1028 (2001).

26 Kato, J. Y., Funa, N., Watanabe, H., Ohnishi, Y. \& Horinouchi, S. Biosynthesis of $\gamma$ butyrolactone autoregulators that switch on secondary metabolism and morphological development in Streptomyces. Proc. Natl Acad. Sci. USA. 104, 2378-2383 (2007).

27 Corre, C., Haynes, S. W., Malet, N., Song, L. \& Challis, G. L. A butenolide intermediate in methylenomycin furan biosynthesis is implied by incorporation of stereospecifically ${ }^{13}$ C-labelled glycerols. Chem. Commun. 46, 4079-4081 (2010).

28 Corre, C. \& Challis, G. L. Evidence for the unusual condensation of a diketide with a pentulose in the methylenomycin biosynthetic pathway of Streptomyces coelicolor A3(2). Chem. Bio. Chem. 6, 2166-2170 (2005).

29 Midland, S. L. et al. The structure of syringolides 1 and 2, novel C-glycosidic elicitors from Pseusdomonas syringae pv. tomato. J. Org. Chem. 58, 2940-2945 (1993).

30 Hopwood, D. A. \& Wright, H. M. Interaction of the plasmid SCP1 with the chromosome of Streptomyces coelicolor A3(2). in Second International Symposium on the Genetics of Industrial Microorganisms (ed. MacDonald, K. D.) 607-619 (Academic Press, London, 1976).

31 Yamasaki, M. \& Kinashi, H. Two chimeric chromosomes of Streptomyces coelicolor $\mathrm{A} 3(2)$ generated by single crossover of the wild-type chromosome and linear plasmid SCP1. J. Bacteriol. 186, 6553-6559 (2004)

32 Vivian, A. \& Hopwood, D. A. Genetic control of fertility in Streptomyces coelicolor A3(2): the IF fertility type. J. Gen. Microbiol. 64, 101-117 (1970).

33 Vivian, A. \& Hopwood, D. A. Genetic control of fertility in Streptomyces coelicolor A3(2): new kinds of donor strains. J. Gen. Microbiol. 76, 147-162 (1973).

34 Hanafusa, T. \& Kinashi, H. The structure of an integrated copy of the giant linear plasmid SCP1 in the chromosome of Streptomyces coelicolor 2612. Mol. Gen. Genet. 231, 363-368 (1992).

35 Yamasaki, M., Redenbach, M. \& Kinashi, H. Integrated structures of the linear plasmid SCP1 in two bidirectional donor strains of Streptomyces coelicolor A3(2). Mol. Gen. Genet. 264, 634-642 (2001).

36 Kinashi, H., Murayama, M., Matsushita, H. \& Nimi, O. Structural analysis of the giant linear plasmid SCP1 in various Streptomyces coelicolor strains. J. Gen. Microbiol., 139, 1261-1269 (1993). 
37 Haneishi, T., Kitahara, N., Takiguchi, Y., Arai, M. \& Sugawara, S. New antibiotics, methylenomycins $\mathrm{A}$ and $\mathrm{B}$. I. Producing organism, fermentation and isolation, biological activities and physical and chemical properties. J. Antibiot. 27, 386-392 (1974).

38 Yamasaki, M., Ikuto, Y., Ohira, A., Chater, K. \& Kinashi, H. Limited regions of homology between linear and circular plasmids encoding methylenomycin biosynthesis in two independently isolated streptomycetes. Microbiology 149, 1351-1356 (2003).

39 Hayakawa, T., Tanaka, T., Sakaguchi, K., Otake, N. \& Yonehara, H. A linear plasmid-like DNA in Streptomyces sp. producing lankacidin group antibiotics. J. Gen. Appl. Microbiol. 25, 255-260 (1979).

40 Hirochika, H. \& Sakaguchi, K. Analysis of linear plasmids isolated from Streptomyces: association of protein with the ends of the plasmid DNA. Plasmid 7, 59-65 (1982).

41 Hirochika, H., Nakamura, K. \& Sakaguchi, K. A linear DNA plasmid from Streptomyces rochei with an inverted terminal repetition of 614 base pairs. EMBO J. 3, 761-766 (1985).

42 Kinashi, H., Mori, E., Hatani, A. \& Nimi, O. Isolation and characterization of linear plasmids from lankacidin-producing Streptomyces species. J. Antibiot. 47, 1447-1455 (1994).

43 Mochizuki, S. et al. The large linear plasmid pSLA2-L of Streptomyces rochei has an unusually condensed gene organization for secondary metabolism. Mol. Microbiol. 48, 1501-1510 (2003).

44 Hiratsu, K., Mochizuki, S. \& Kinashi, H. Cloning and analysis of the replication origin and the telomeres of the linear plasmid PSLA2-L in Streptomyces rochei. Mol. Gen. Genet. 263, 1015-1021 (2000).

45 Arakawa, K., Mochizuki, S., Yamada, K., Noma, T. \& Kinashi, H. $\gamma$-Butyrolactone autoregulator-receptor system involved in lankacidin and lankamycin production and morphological differentiation in Streptomyces rochei. Microbiology 153, 1817-1827 (2007).

46 Horinouchi, S. \& Beppu, T. Hormonal control by A-factor of morphological development and secondary metabolism in Streptomyces. Proc. Jpn. Acad. Ser. B 83, 277-295 (2007).

47 Yamamoto, S., He, Y., Arakawa, K. \& Kinashi, H. $\gamma$-Butyrolactone-dependent expression of the SARP gene srrY plays a central role in the regulatory cascade leading to lankacidin and lankamycin production in Streptomyces rochei. J. Bacteriol. 190, 1308-1316 (2008)

48 Suzuki, T., Mochizuki, S., Yamamoto, S., Arakawa, K. \& Kinashi, H. Regulation of lankamycin biosynthesis in Streptomyces rochei by two SARP genes, srrY and srrZ. Biosci. Biotechnol. Biochem. 74, 819-827 (2010).

49 Arakawa, K., Sugino, F., Kodama, K., Ishii, T. \& Kinashi, H. Cyclization mechanism for the synthesis of macrocyclic antibiotic lankacidin in Streptomyces rochei. Chem. Biol. 12, 249-256 (2005).

50 Tatsuno, S., Arakawa, K. \& Kinashi, H. Analysis of the modular-iterative mixed biosynthesis of lankacidin by heterologous expression and gene fusion. J. Antibiot. 60, 700-708 (2007).

51 Tatsuno, S., Arakawa, K. \& Kinashi, H. Extensive mutational analysis of modulariterative mixed polyketide biosynthesis of lankacidin in Streptomyces rochei. Biosci. Biotechnol. Biochem. 73, 2712-2719 (2009).

52 Arakawa, K., Kodama, K., Tatsuno, S., Ide, S. \& Kinashi, H. Analysis of the loading and hydroxylation steps in lankamycin biosynthesis in Streptomyces rochei. Antimicrob. Agents Chemother. 50, 1946-1952 (2006).

53 Arakawa, K., Suzuki, T. \& Kinashi, H. Gene disruption analysis of the two glycosylation steps in lankamycin biosynthesis in Streptomyces rochei. Actinomycetol. 22, 35-41 (2008).

54 Auerbach, T. et al. The structure of ribosome-lankacidin complex reveals ribosomal sites for synergistic antibiotics. Proc. Natl Acad. Sci. USA, 107, 1983-1988 (2010).

55 Ward, J. M. \& Hodgson, J. E. The biosynthetic genes for clavulanic acid and cephamycin production occur as a 'super-cluster' in three Streptomyces. FEMS Microbiol. Lett. 110, 239-242 (1993).
56 Pérez-Llarena, F. J., Liras, P., Rodríguez-García, A. \& Martín, J. F. A regulatory gene (ccaR) required for cephamycin and clavulanic acid production in Streptomyces clavuligerus: amplification results in overproduction of both $\beta$-lactam compounds. J. Bacteriol. 179, 2053-2059 (1997).

57 Migita, A. et al. Identification of a gene cluster of polyether antibiotic lasalocid from Streptomyces lasaliensis. Biosci. Biotechnol. Biochem. 73, 169-176 (2009).

58 Shichijo, Y. et al. Epoxide hydrolase Lsd19 for polyether formation in the biosynthesis of lasalocid A: direct experimental evidence on polyene-polyepoxide hypothesis in polyether biosynthesis. J. Am. Chem. Soc. 130, 12230-12231 (2008).

59 Watanabe, K. et al. Total biosynthesis of antitumor nonribosomal peptides in Escherichia coli. Nat. Chem. Biol. 2, 423-428 (2006).

60 Gravius, B. et al. The $387 \mathrm{~kb}$ linear plasmid pPZG101 of Streptomyces rimosus and its interactions with the chromosome. Microbiology 140, 2271-2277 (1994).

61 Pandza, S. et al. Recombination between the linear plasmid pPZG101 and the linear chromosome of Streptomyces rimosus can lead to exchange of ends. Mol. Microbiol. 28, 1165-1176 (1998).

62 Smith, D. J. et al. $\beta$-Lactam antibiotic biosynthetic genes have been conserved in clusters in prokaryotes and eukaryotes. EMBO J. 9, 741-747 (1990).

63 Kinashi, H., Doi, M. \& Nimi, O. Isolation of large linear plasmids from $\beta$-lactamproducing actinomycete strains. Biotechnol. Lett. 17, 243-246 (1995).

64 Netolitzky, D. J., Wu, X., Jensen, S. E. \& Roy, K. L. Giant linear plasmids of $\beta$-lactam antibiotic producing Streptomyces. FEMS Microbiol. Lett. 131, 27-34 (1995).

$65 \mathrm{Wu}$, X. \& Roy, K. L. Complete nucleotide sequence of a linear plasmid from Streptomyces clavuligerus and characterization of its RNA transcripts. J. Bacteriol. 175, 37-52 (1993).

66 Wu, W., Leblanc, S. K., Piktel, J., Jensen, S. E. \& Roy, K. L. Prediction and functional analysis of the replication origin of the linear plasmid pSCL2 in Streptomyces clavuligerus. Can. J. Microbiol. 52, 293-300 (2006).

67 Medema, M. H. et al. The sequence of a $1.8-\mathrm{Mb}$ bacterial linear plasmid reveals a rich evolutionary reservoir of secondary metabolic pathways. Genome Biol. Evol. 2, 212-224 (2010).

68 Zotchev, S. B., Soldatova, L. I., Orekhov, A. V. \& Schrempf, H. Characterization of a linear extrachromosomal DNA element (pBL1) isolated after interspecific mating between Streptomyces bambergiensis and S. lividans. Res. Microbiol. 143, 839-845 (1992).

69 Evans, M., Kaczmarek, F. S., Stutzman-Engwall, K. \& Dyson, P. Characterization of a Streptomyces-lividans-type site-specific DNA modification system in the avermectinproducer Streptomyces avermitilis permits investigation of two novel giant linear plasmids, pSA1 and pSA2. Microbiology 140, 1367-1371 (1994).

$70 \mathrm{Liu}, \mathrm{W}$. et al. The neocarzinostatin biosynthetic gene cluster from Streptomyces carzinostaticus ATCC15944 involving two iterative type I polyketide synthase. Chem. Biol. 12, 293-302 (2005).

71 Tang, G. L., Cheng, Y. Q. \& Shen, B. The biosynthetic gene cluster of the antitumor antibiotic leinamycin from Streptomyces atroolivaceus S-140 revealing unprecedented architectural complexity for a hybrid nonribosomal peptide synthetase-polyketide synthase. Chem. Biol. 11, 33-45 (2004)

72 Liu, W., Christenson, S. D., Standage, S. \& Shen, B. Biosynthesis of the enediyne antitumor antibiotic C-1027. Science 297, 1170-1173 (2002).

73 Ravel, J., Wellington, E. M. \& Hill, R. T. Interspecific transfer of Streptomyces giant linear plasmids in sterile amended soil microcosms. Appl. Environ. Microbiol. 66, 529-534 (2000).

74 Huang, C.H. et al. Linear plasmid SLP2 of Streptomyces lividans is a composite replicon. Mol. Microbiol. 47, 1563-1576 (2003).

75 Choulet, F. et al. Evolution of the terminal regions of the Streptomyces linear chromosome. Mol. Biol. Evol. 23, 2361-2369 (2006).

76 Sakaguchi, K. Invertrons, a class of structurally and functionally related genetic elements that includes linear DNA plasmids, transposable elements, and genomes of adeno-type viruses. Microbiol Rev. 54, 66-74 (1990). 